\title{
Joint Discussion 15 New cosmology results from the Spitzer Space Telescope
}

\author{
George Helou and David T. Frayer (eds.) \\ Spitzer Science Center, California Institute of Technology, Pasadena, CA 91125, USA \\ email: gxh@ipac.caltech.edu
}

\begin{abstract}
We present and discuss recent new results from the Spitzer Space Telescope and their impact on cosmology.
\end{abstract}

Keywords. cosmology: observations, large-scale structure of universe

\section{Introduction}

Joint Discussion 15 reviewed results from the Spitzer Space Telescope and their impact on cosmology, and sought to stimulate a synthesis between them and corresponding recent results from increasingly powerful X-ray, radio, optical, near-infrared and $\gamma$-ray observatories. By bringing together experts from all these fields we hoped to formulate new approaches to the following questions.

- How do the currently known galaxy populations emerge and evolve?

- What is the relationship of the infrared populations at high redshift to the populations in the local universe?

- How does the ultraviolet/optical-based star formation history compare to the history derived from infrared studies?

- How is the rising abundance of heavy chemical elements over time related to the history of galaxy formation and evolution?

- What is the contribution of Active Galactic Nuclei (AGN) to the luminosity of these populations, and how does that contribution evolve?

- What is the role of a starburst phase or a heavy accretion phase in the life cycle of individual galaxies?

- What is the nature of the sources detectable in the X-Rays and infrared, but not the ultraviolet/optical, and what other new populations of objects emerge from the new surveys?

During the introductory session, Tom Soifer, Director of the Spitzer Science Center reported on the status of the Spitzer Observatory, and gave an overview of the Spitzer Science Program related to Cosmology.

\section{The modern universe}

Seb Oliver presented a multi-wavelength view of the universe at $z<1$, providing the scientific motivation for relatively shallow surveys of large areas on the sky, such as the Spitzer Wide-area Infrared Extragalactic Survey (SWIRE, Lonsdale et al. 2004). Such surveys result in a wide variety of objects, challenging the population models to account for all of them (Lagache et al. 2004; Rowan-Robinson et al. 2005). Davoodi et al. (2006) compared SWIRE detections to Sloan Digital Sky Survey (SDSS) detections, looking in 
particular for the infrared properties of galaxies in the context of their blue sequence or red sequence classification. They found that $18 \%$ of their objects (195 of 1114) have red optical colors and $24 \mu \mathrm{m}$ excess, reflecting either AGN or enhanced star formation activity, suggesting this activity is superposed on a bulge-dominated system.

Rob Kennicutt reported on progress in the study of galaxies in the local universe, highlighting a better characterization of the integrated spectral energy distributions from the ultraviolet to the infrared (Dale et al. 2007), combining data from GALEX (Galaxy Evolution Explorer) and Spitzer; new robust star formation indicators combining $\mathrm{H} \alpha$ and $24 \mu \mathrm{m}$ emission from galaxies (Calzetti et al. 2007; Kennicutt et al. 2007); and a careful analysis of variability in the Aromatic Feature emission in the range $5 \mu \mathrm{m}<\lambda<20 \mu \mathrm{m}$ (Smith et al. 2007).

Vassilis Charmandaris and Jim Houck summarized the state of knowledge on dust properties in low metallicity environments, best advanced by the study of nearby dwarf galaxies. The existence of a threshold in metallicity below which Aromatic Feature emission is clearly suppressed is now well established (Engelbracht et al. 2005; Smith et al. 2007). However, the underlying causes for this deficiency and other contributing factors are still open to discussion (Rosenberg et al. 2006; Draine et al. 2007).

Eckhard Sturm reviewed new results on Ultra-Luminous Infrared Galaxies (ULIRG) and AGN in the local universe, stressing the diversity in their spectra and showing that these classes display evidence for emission associated with both star formation and black hole heating, often modified by strong extinction in dense interstellar medium surrounding the power source (Armus et al. 2007; Weedman et al. 2006); this high extinction often reveals crystals and ices on very cold grains (Spoon et al. 2006). Silicate emission has now been clearly detected for the first time in AGN. It is also becoming clear from the study of galaxies with both AGN and star formation heating diagnostics like ratios of Aromatic Feature emission or fine-structure line emission can be modified by factors other than the presence of AGN, and should therefore be interpreted with caution (Sturm et al. 2006; Dale et al. 2006).

\section{The evolving universe}

Emanuele Daddi considered what we are learning from Spitzer about stars and star formation in the universe at a redshift $z \simeq 2$. Substantial insight into the properties of stellar populations of galaxies at these distances are yielded by studies in the 3 to $8 \mu \mathrm{m}$ range using the IRAC instrument on Spitzer. Maraston et al. (2006) demonstrate the importance of including all phases of stellar evolution in the population models, in particular the thermally pulsing asymptotic giant branch, in order to reproduce the Spitzer-IRAC data. One result of this stellar population investigation is that most massive galaxies $\left(\sim 10^{11} \mathrm{M}_{\odot}\right)$ observed at $z \simeq 2$ are also bright in the infrared and qualify as Ultra-Luminous Infrared Galaxies, whereas massive but relatively inactive galaxies are rare (Daddi et al. 2005).

Ranga-Ram Chary discussed dusty galaxies as seen by Spitzer in the deepest $24 \mu \mathrm{m}$ surveys, echoing the result that red, massive galaxies at redshifts $1<z<3.5$ have a specific star formation rate an order of magnitude greater than their analogs at redshifts $0.3<z<0.75$, suggesting that the bulk of star formation in massive galaxies occurs at early cosmic epochs and is largely complete by $z \simeq 1.5$ (Papovich et al. 2006). The spectral energy distributions of galaxies at high redshifts appear consistent with those of galaxies in the local universe at similar luminosity given available data; with that assumption, 
the cosmic infrared background can be reproduced if one includes all sources down to $f_{\nu}(24 \mu \mathrm{m})=24 \mu \mathrm{Jy}$, with AGN contributing a small fraction of the total background.

David Frayer reviewed results from deep and ultra-deep far-infrared surveys with Spitzer at 70 and $160 \mu \mathrm{m}$. The infrared-radio correlation seems to hold for galaxies out to redshifts $z \simeq 1$ (e.g., Appleton et al. 2004). Source counts have been measured down to $f_{\nu}(70 \mu \mathrm{m})=1.2 \mathrm{mJy}$, accounting for about $60 \%$ of the cosmic infrared background (Frayer et al. 2006). The 70-24 $\mu \mathrm{m}$ colors suggest that galaxies are on average cooler at $z \simeq 1$ than expected from the luminosity distribution at that epoch.

Guilaine Lagache spoke to results obtained from spectroscopic studies of high-redshift infrared-luminous galaxies using Spitzer. While the interpretation of these spectra is still evolving rapidly, it is pretty clear that trends identified in the local universe continue to apply at $z \simeq 1-2$, such as the greater contribution of AGN to powering sources as the source luminosity rises (Yan et al. 2007). However, the potential existence of galaxies at $L_{\mathrm{IR}} \simeq 10^{13} \mathrm{~L}_{\odot}$ which are energetically dominated by star formation is unique to high redshifts.

Pieter van Dokkum addressed the nature of massive galaxies at redshifts 2 to 3 , with an emphasis on the multiplicity of techniques to identifying such objects, and approaches to constructing samples that avoid the biases inherent to each technique. With the nearinfrared bump shifting to longer wavelengths, Spitzer is clearly at an advantage in identifying such objects, though inconsistencies remain among various authors regarding the fraction of massive galaxies at $z \simeq 2-3$ which appear completely quiescent (e.g., Kriek et al. 2006).

\section{The distant universe}

Tommy Wiklind examined how Spitzer has helped identify and study objects whose optical emission is redshifted into the mid-infrared, and in particular galaxies at $z>5$. Building on the early detection of a massive post-starburst galaxy at $z \simeq 6.5$ (Mobasher et al. 2005), the GOODS (Great Observatories Origins Deep Survey) team has identified 18 candidates for similar objects at $z>5$, using Spitzer data as the key. The objects average $\sim 2 \times 10^{11} \mathrm{M}_{\odot}$ and run between 0.2 and $1 \mathrm{Gyr}$ in age, with little or no on-going star formation, and modest or low extinction. They must have formed at $z>6$ and possibly as high as $z \simeq 25$, and are observed with a density $4-5 \times 10^{-5} \mathrm{Mpc}^{-3}$ that starts to challenge the predictions of $\Lambda$ CDM models of galaxy formation (Somerville 2005).

Xiaohui Fan reviewed studies of quasars at high redshifts, where Spitzer can detect and study accretion disk and dust emission, helping to find $z>5$ quasars with a frequency of 1 per 1.5 sq.deg, greater than optical ground-based surveys (Cool et al. 2006). These quasars are also among the least luminous known at these redshifts, and appear similar in their spectral energy distribution to lower redshift objects, adding to the evidence for a lack of strong evolution in the properties of quasars. While $z \simeq 6$ quasars tend to show prominent hot dust emission, the origin of that dust remains uncertain, especially that some objects probably have unusual dust properties (Jiang et al. 2006).

Richard Ellis summarized searches for the sources responsible for cosmic re-ionization, where Spitzer plays a key role because of its ability to fill in the spectrum beyond the longest wavelength available from the ground. The follow-up of $i^{\prime}$-band drop-out sources for instance reveals that $z \simeq 6$ sources have dominant stellar populations with ages $>100 \mathrm{Myr}$, and a history of vigorous star formation at $z>7$. The progenitors of 
such systems must have allowed star formation to play a prominent role in reionizing the universe (Eyles et al. 2005).

Anton Koekemoer presented joint Spitzer/HST/Chandra results on Extreme X-ray / Optical sources (EXOs), defined by their large ratio of X-ray to visible flux. EXOs are readily detected in the near-infrared and by Spitzer in the mid-infrared. While many EXOs reside at $2<z<5$ and exhibit high optical depth or low optical depths and active star formation, at least one candidate remains best explained as a $z>7$ AGN with a power law spectrum and excess emission beyond $10 \mu \mathrm{m}$ (Koekemoer et al. 2005). There are no obvious local universe counterparts to EXOs.

\section{Integrated perspectives}

Marijn Franx offered an overview of the evolution of galaxies measured by Spitzer and multi-wavelength surveys, emphasizing the two powerful tools offered by Spitzer: imaging and photometry at $3-8 \mu \mathrm{m}$ to trace the evolution of stellar mass over cosmic time scales, and at $24 \mu \mathrm{m}$ to trace the star formation history. While clear patterns of decline in the cosmic star formation rate are detected (e.g., Bell et al. 2005), a remaining limitation is the translation of $24 \mu \mathrm{m}$ measurements into bolometric luminosities, critical for estimating the star formation rate. Studies combining Spitzer data with submillimeter $850 \mu \mathrm{m}$ and radio $20 \mathrm{~cm}$ data suggest a downward revision of the amount of star formation at higher redshifts, and half the total star formation occurring since $z \simeq 1.4$ (Wang et al. 2006).

Mark Dickinson discussed the state of our understanding of the global history of star formation in the Spitzer era, again underscoring the essential need for multi-wavelength studies, and the importance not only of estimating properly bolometric luminosities for individual objects, but also of integrating over populations when only the most luminous objects are detected. Multi-wavelength studies are unveiling new populations, such as AGN undetected at X-rays but evident in radio and infrared data (e.g., Donley et al. 2005), or Ultra-Luminous Infrared Galaxies with cooler far-infrared colors than is common in the local universe (e.g., Pope et al. 2006).

Carlos Frenk described the state of the art in interpreting Spitzer data in the context of Cold Dark Matter numerical simulations of cosmic history. While the models still manage to explain Spitzer data, they are strained in their ability to represent Aromatic Feature emission properties, and they require modifications to the Initial Mass Function of stars, direct evidence for which is sparse (e.g., Granato et al. 2004).

Jean-Loup Puget reviewed the relation between the extragalactic background light and the star formation history, pointing out the critical role Spitzer plays in elucidating the rise of dust, which converts at least half the visible photons into infrared photons. Of particular interest is the rise of Aromatic compounds, which require cycling of material from stellar outflows through dense molecular clouds then out into photo-dissociation regions, going from simple carbon compounds to aliphatic then aromatic compounds. The infrared background is clearly consistent with source counts in the mid-infrared including $24 \mu \mathrm{m}$, but only indirectly related to source counts at far-infrared to submillimeter bands (e.g., Dole et al. 2006).

Sylvain Veilleux discussed Ultraluminous Infrared Galaxies in the context of cosmic evolution, stressing in particular their likely role as progenitors of spheroids, and their likely central role in black hole growth. In addition, these objects probably dominate the enrichment of the intergalactic medium, because of their over-abundance at epochs $z>2$ 
and their tendency to generate super-winds that disperse supernova ejecta well beyond their halos.

Thorsten Naab reported on recent progress in understanding galaxy mergers, a key process in the evolution of the universe. Current models reproduce quite well the mass interactions, but more work is needed to represent more accurately the star formation activity triggered in close encounters and mergers.

\section{Conclusions}

In his concluding remarks, Simon Lilly described the impact of Spitzer on our understanding of the high redshift universe as enormous, fulfilling the most optimistic expectations. He highlighted the value of Legacy Science projects, pioneered by Spitzer, and their key contribution to a timely diffusion and assimilation of the scientific returns from the observatory. Looking ahead, he advocated the need for 'a billion galaxy survey', achievable by going to $I_{\mathrm{AB}} \simeq 25 \mathrm{mag}$ over a large area of the sky, perhaps $10^{4}$ square degrees. A critical component of such an undertaking would be a survey at 3.6 and $4.5 \mu \mathrm{m}$ during the Spitzer warm mission reaching a commensurate depth, and requiring by itself several years of the warm mission.

\section{Scientific Organizing Committee}

Jacqueline A. Bergeron (France), Catherine J. Cesarsky (Germany), Vassilis Charmandaris (Greece), Thierry J-L. Courvoisier (Switzerland), George Helou (USA, chair), Myungshin Im (South Korea), Leopoldo Infante (Chile), Robert J. Ivison (UK), Haruyuki Okuda (Japan), Jan Palous (Czech Republic), Charles C. Steidel (USA), and Rasjid A. Sunyaev (Russian Federation).

\section{Acknowledgements}

We would like to acknowledge the members of the Science Organizing Committee. NASA, through the Spitzer Science Center, provided travel support for several of the speakers. The Spitzer Space Telescope is operated by the Jet Propulsion Laboratory, California Institute of Technology under contract with NASA.

\section{References}

Appleton, P. N., Fadda, D. T., Marleau, F. R., et al. 2004, ApJS, 154, 147

Armus, L., Charmandaris, V., Bernard-Salas, J., et al. 2007, ApJ, 656, 148

Bell, E. F., Papovich, C., Wolf, C., et al. 2005, ApJ, 625, 23

Calzetti, D., Kennicutt, R. C., Engelbracht, C. W., et al. 2007, ApJ, 666, 870

Cool, R. J., Kochanek, C. S.; Eisenstein, D., et al. 2006, AJ, 132, 823

Daddi, E., Dickinson, M., Chary, R., et al. 2005, ApJ (Letters), 631, L13

Dale, D. A., Smith, J. D. T., Armus, L., et al. 2006, ApJ, 646, 161

Dale, D. A., Gil de Paz, A., Gordon, K. D., et al. 2007, ApJ, 655, 863

Davoodi, P., Pozzi, F., Oliver, S., et al. 2006, MNRAS, 371, 1113

Dole, H., Lagache, G., Puget, J.-L., et al. 2006, A\&A 451, 417

Donley, J. L., Rieke, G. H., Rigby, J. R., \& Pèrez-González, P. G. 2005, ApJ, 634, 169

Draine, B. T., Dale, D. A., Bendo, G., et al. 2007, ApJ, 663, 866

Engelbracht, C. W., Gordon, K. D., Rieke, G. H., et al. 2005, ApJ, 628, 29

Eyles, L. P., Bunker, A. J., Stanway, E. R., et al. 2005, MNRAS, 364, 443

Frayer, D. T., Huynh, M. T., Chary, R., et al. 2006, ApJ (Letters), 647, L9

Granato, G. L., De Zotti, G., Silva, L., et al. 2004, ApJ, 600, 580 
Jiang, L., Fan, X., Hines, D. C., et al. 2006, AJ, 132, 2127

Kennicutt, R. C., Calzetti, D., Walter, F., et al. 2007, ApJ, in press [2007arXiv0708.0922K]

Koekemoer, A. M., Alexander, D. M., Bauer, F. E., et al. 2005, in: A. Wilson (ed), The Dusty and Molecular Universe: A Prelude to Herschel and ALMA, ESA SP-577, p. 111

Kriek, M., van Dokkum, P. G., Franx, M., et al. 2006, ApJ (Letters), 649, L71

Lagache, G., Dole, H., Puget, J.-L., et al. 2004, ApJS, 154, 112

Lonsdale, C., Polletta, M.d.C., Surace, J., et al. 2004, ApJS, 154, 54

Maraston, C., Daddi, E., Renzini, A., et al. 2006, ApJ, 652, 85

Mobasher, B., Dickinson, M., Ferguson, H. C., et al. 2005, ApJ, 635, 832

Papovich, C., Moustakas, L. A., Dickinson, M., et al. 2006, ApJ, 640, 92

Pope, A., Scott, D., Dickinson, M., et al. 2006, MNRAS 370, 1185

Rowan-Robinson, M., Babbedge, T., Surace, J., et al. 2005, AJ, 129, 1183

Rosenberg, J. L., Ashby, M. L. N., Salzer, J. J., Huang, J.-S. 2006, ApJ 636, 742

Smith, J. D. T., Draine, B. T., Dale, D. A., et al. 2007, ApJ, 656, 770

Somerville, R. S. 2005, in: A. Renzini, \& R. Bender (eds.), Multiwavelength Mapping of Galaxy Formation and Evolution, Proc. ESO Workshop, Venice, Italy, 13-16 October 2003 (Berlin: Springer), p. 131

Spoon, H. W. W., Tielens, A. G. G. M., Armus, L., et al. 2006, ApJ, 638, 759

Sturm, E., Hasinger, G., Lehmann, I., et al. 2006, ApJ, 642, 81

Wang, W.-H., Cowie, L. L., \& Barger, A. J. 2006, ApJ, 647, 74

Weedman, D. W., Soifer, B. T., Hao, L., et al. 2006, ApJ, 651, 101

Yan, L., Sajina, A., Fadda, D., Choi, P., Armus, L., Helou, G., et al. 2007, ApJ, 658, 778 